\title{
Un passé publicitaire à la mode générationnelle
}

The Generational Past of Advertising

Myriam Bahuaud et Agnès Pecolo

\section{(2) OpenEdition}

Journals

Édition électronique

URL : http://journals.openedition.org/edc/7918

DOI : 10.4000/edc.7918

ISSN : 2101-0366

Éditeur

Université de Lille

Édition imprimée

Date de publication : 1 décembre 2018

Pagination : 21-36

ISBN : 978-2-917562-20-8

ISSN : $1270-6841$

Référence électronique

Myriam Bahuaud et Agnès Pecolo, « Un passé publicitaire à la mode générationnelle », Études de communication [En ligne], 51 | 2018, mis en ligne le 01 janvier 2021, consulté le 11 février 2021. URL: http://journals.openedition.org/edc/7918; DOI : https://doi.org/10.4000/edc.7918 


\section{Un passé publicitaire à la mode générationnelle \\ The Generational Past of Advertising}

Myriam Bahuaud

Maîtres de conférences en Sciences de l'Information

et de la Communication

myriam.bahuaud@u-bordeaux-montaigne.fr

Agnès Pecolo

Université Bordeaux Montaigne,

MICA (Médiation, Information, Communication, Art ; EA 4426)

agnes.pecolo@u-bordeaux-montaigne.fr 
Afin d'exprimer tout à la fois un ancrage dans le passé et une continuité entre passé, présent et futur, les marques trient dans leur héritage et réaniment des éléments de leur patrimoine, garants d'authenticité. À partir d'un corpus de publicités télévisées contemporaines qui opèrent une reprise de campagnes de communication antérieures, nous analysons, par le prisme de l'approche générationnelle, les mises en scène visuelle et discursive, et la mise en forme de I'articulation passé/présent/futur. Les entrées authenticité et nostalgie sont les terreaux favoris de cette forme de communication publicitaire.

Mots-clés : authenticité, nostalgie, patrimoine, communication publicitaire et générationnelle.
Commercial brands seeking to express an anchoring in the past and a sense of continuity between past, present and future, select and revive elements of their heritage as guarantees of authenticity. In this article we analyze a corpus of contemporary television commercials which reuse previously aired communication campaigns. We explore, by means of a generational approach, visual and discursive staging as well as the links created between past, present and future. Authenticity and nostalgia are the preferred basis of this form of advertising communication.

Keywords: authenticity, nostalgia, heritage, advertising communication, generational communication. 
Si l'utilisation du patrimoine de marque n'est pas neuve (Pecot et De Barnier, 2017), pas plus que celle du « c'était mieux avant » - que l'on pourrait nommer "le syndrome du rétroviseur " (Nussbaum et Evéquoz, 2014) - il semble en revanche que nous baignons aujourd'hui dans une époque particulièrement tentée par les retours en arrière et tournée vers hier. Les changements angoissent et la bascule de notre société dans un nouveau monde aux contours encore flous alimente une représentation du passé aussi glorifiée que celle de l'avenir est brouillée (Angé, 2015 ; Hartog, 2003). Les artefacts et pratiques publicitaires, parce qu'ils « [...] se transforment et se renouvellent au fil du temps en s'adaptant aux mutations économiques, sociales et technologiques " (De lulio, 2016, 101), n'échappent pas à ce penchant pour les racines et les sentiments nostalgiques. Depuis le début des années 2000, exploiter les fonds du passé, instrumentaliser des catalogues de productions culturelles pour réveiller des souvenirs nostalgiques, semblent être une composante intrinsèque de la culture de masse et de l'industrie des médias (Reynolds, 2012). Afin d'exprimer tout à la fois un ancrage dans le passé et une continuité entre passé, présent et futur, les marques vont trier dans leur « corporate heritage " (Urde et al., 2007) et réanimer des éléments de leur patrimoine (un passé amélioré et sélectif) garants d'authenticité, tels que des savoir-faire, la figure d'un fondateur, des archives, des produits cultes ou encore d'anciennes publicités. C'est sur ces dernières que nous nous concentrons ici avec pour objectif de mettre à jour différentes manières pour les marques de combiner présence dans le passé et inscription dans le présent. Quelles mises en scène, en mots, en sons et en images est-il possible de repérer? Comment cette articulation passé/présent/ futur prend forme au sein des publicités ? Nous souhaitons, par le prisme de l'approche générationnelle (Pecolo et Bahuaud, 2016), éclairer les procédés publicitaires à l'œuvre et les différentes mises en forme communicationnelles choisies quand les marques décident d'utiliser le rétroviseur et optent pour une méthode qui consiste à mettre au goût du jour leurs produits tout en pointant le fait qu'ils ne sont pas nés d'hier.

Après une explicitation méthodologique, notre développement se structurera en deux parties. La première décryptera les communications de marques à capital historique qui usent, comme nous le verrons, des codes de l'authenticité mais aussi et surtout de ceux de la nostalgie. La deuxième partie se focalisera sur les différents procédés sonores, visuels et discursifs réanimés par les marques dans l'espoir de déclencher des sentiments nostalgiques.

1.

\section{Spots télévisuels et registres générationnels}

Afin de dégager les modalités d'usage du passé dans les publicités contemporaines, et plus spécifiquement celles qui opèrent une reprise de campagnes de communication antérieures, nous avons constitué un corpus de spots télévisuels que nous avons analysés par le prisme de l'approche générationnelle. 


\subsection{La constitution du corpus}

L'élaboration du corpus s'est opérée sur la base de deux terrains de chasse complémentaires : une veille sur l'actualité publicitaire mise en place depuis $2014^{1}$ qui a permis de repérer des marques entrant dans notre champ d'étude, croisée à la (re)lecture d'un corpus de spots télévisés ${ }^{2}$ issu d'une recherche antérieure (Pecolo et Bahuaud, 2016) et dans lequel nous avons pu sélectionner des spots intéressants pour notre approche en ce qu'ils mettaient en scène leur propre passé publicitaire.

Travailler à partir de matériaux émanant de publications professionnelles dans les secteurs de la communication et du marketing induit deux remarques quant aux précautions à prendre pour l'analyse. En premier lieu, il s'agit de souligner la particularité de l'information diffusée dans ces supports. Cette presse, lue sur le lieu de travail, s'adresse à travers sa vocation utilitaire, aux présidents, directeurs généraux et directeurs marketing d'entreprises, d'agences de communication et de médias. II faut donc, en analysant les résultats, garder en mémoire non seulement l'interdépendance entre les journalistes-rédacteurs et leurs lecteurs, professionnels de la communication mais également les liens étroits avec différents types d'acteurs économiques du secteur d'activités. Ces derniers, qualifiés par Fabien Ohl de " socio-marketers " (2002) ou encore de " sociologues de la consommation », de "marketo-sociologues » voire de " propagateurs " par Bernard Cova, Marie Louyot-Gallicher et Audrey Bonnemaizon $(2010,39)$, forment une petite communauté d'« experts » très souvent interpellée dans la presse étudiée. À travers cette auto-promotion professionnelle, les newsletters et sites de notre veille contribuent à la construction de discours sociaux sur la nostalgie, l'authenticité et la patrimonialisation des marques, et à la construction d'une figure d'un consommateur qu'ils présentent comme régressif et/ou nostalgique de son enfance. En second lieu, nous travaillons sur des marques relayées par le secteur professionnel, sans prendre en compte ni les critères d'appréciation de la part des publics ni les indicateurs de diffusion et encore moins les indicateurs de performance marketing et/ou communicationnelle. II ne s'agit pas de retirer de notre analyse une quelconque exploitation managériale mais bien d'avoir un regard critique et d'appréhender la publicité «[...] comme une forme de construction sociale de la réalité qui contribue à renforcer ou, en tout cas, à orienter les valeurs et les symboles dont elle se sert »(De lulio, 2016, 99).

14 newsletters et 4 sites : @marketing (webactu de Marketing Magazine) - ADN (webactu du magazine Influencia) - Le 13 hOO de la com' (webactu du magazine Stratégies) - CBNews la Matinale (webactu de la revue CBNews) - ilétaitunepub.fr - lemondemarketing.com - laréclame.fr et marketing-et-communication.fr

2292 spots diffusés de janvier 2012 à février 2013 sur les chaînes de télévision françaises, horaires et chaînes confondus. 
Le croisement de nos deux terrains nous a permis de constituer, dans un premier temps, un corpus de 60 marques appartenant à différents secteurs ${ }^{3}$ qui, toutes, mobilisent les temps biographique et historique.

Le cadre de cet article et la thématique du dossier centrée sur la réactualisation des artefacts publicitaires du passé ne se prêtent pas à l'exposé détaillé des résultats. Ces derniers relèvent des pratiques diverses telles que la référence à une époque passée (via la musique ou un symbole culturel et/ ou médiatique), un argumentaire centré sur l'authenticité, une campagne rétro, voire anti ou " pseudo » rétro, et enfin des sagas publicitaires. Nous entendons par sagas publicitaires des campagnes reprenant le même thème, personnage, symbole, etc. déclinées dans un temps long, à savoir faisant appel aux publicités des décennies précédentes. Ces sagas, au nombre de seize dans notre corpus, sont présentes sur uniquement quatre secteurs sur les dix répertoriés, et douze appartiennent au secteur alimentaire. Aussi nous nous centrons ici sur un corpus restreint de dix marques du secteur alimentaire, particulièrement intéressantes quant à leur choix de réanimer d'anciennes publicités télévisuelles. Ces dix marques retenues sont par ordre alphabétique : Caprice des Dieux (1956), Crunch (1960), Hollywood (1972), Les Produits Laitiers (1963, publicités créées par le CNIEL Centre National Interprofessionnel de I'Economie Laitière), Lesieur (1908), Lustucru (1911), Mousline (1963), Panzani (1950), Petit Écolier (1983) et Puget (1857). Nous évoquerons ici les différents usages de leurs artefacts publicitaires du passé en repérant les symboles constitutifs d'un patrimoine de marque visuels, sonores et discursifs reliant les spots contemporains et les " anciens ". Nous avons pu constater, selon les marques, une variété de procédés que nous avons analysés par le prisme de l'approche générationnelle.

1.2. L’approche générationnelle mobilisée : cycles de vie des produits et des individus

Le choix de cette approche nous permet de repérer, dans les stratégies publicitaires, cinq registres communicationnels liés aux âges de la vie et qui jouent avec le temps et les générations (Pecolo et Bahuaud, 2016). Parmi eux, trois registres ${ }^{4}$ - générationnel, transgénérationnel et trans'âges - sollicitent les cycles de vie des personnes et des produits. Ainsi, ils permettent une traversée des âges et des générations et par là, une plongée dans I'histoire commune des marques et des individus. Une communication générationnelle se demande auprès de quels jeunes et quels vieux la marque doit communiquer afin de jouer avec leurs marqueurs générationnels. Il s'agit donc, pour les communicants, d'opérer des détours par les profils culturels des générations. Une com-

3 Alimentaire (24), boissons (8), automobiles (5), distribution (5), médias/technologies (5), habillement/chaussures (4), Ameublement (2), cosmétiques/ hygiène (3), voyage/tourisme (2), autres (2 : Bancaire, Energie).

4 Les deux autres registres, non évoqués ici, sont liés à une communication d'âge et à une communication intergénérationnelle. 
munication transgénérationnelle convoque la traversée des générations avec un produit consommé au fil des générations. Une communication trans'âges mobilise la traversée des stades de vie d'un individu (ou d'une famille) qui, au fil des âges, a grandi aux côtés du produit et/ou de la marque. Les mises en scène publicitaires peuvent donc jouer avec plusieurs registres. Elles font écho, par un registre transgénérationnel, au cycle de vie d'un produit qui traverse les générations et le temps; l'idée étant de démontrer qu'elles peuvent perdurer. Elles accompagnent également des individus à travers leur cycle de vie, illustrant ainsi, par un registre trans'âges, qu'ils grandissent avec la marque ou le produit. Nous retrouvons ce dernier registre dans notre corpus avec les « Petit Écolier». Dans une publicité de 2013, des employés jouent dans leur bureau et, à $16 \mathrm{hoO}$ précises, dégustent ces gâteaux de Lu en organisant un circuit ludique grâce aux accessoires de bureau tout en narguant des enfants en cour de récréation en face de l'entreprise. La marque use du registre trans'âges à travers la mise en scène d'un goûter pour enfant qui plaît aux grands, dans une version de juvénilisation adulte. Intitulé "La riposte ", ce spot est un miroir des premières versions des années 1990 sur lesquelles nous reviendrons en dernière partie.

Pour chacune des marques présentées ci-dessus, nous avons produit une grille d'analyse. Elle s'intéresse - conformément aux objectifs évoqués - à la façon dont chaque marque a réactivé des artefacts publicitaires du passé. Cette grille permet de catégoriser chaque artefact présent dans une ou plusieurs publicités contemporaines (qu'il soit sonore, visuel ou discursif) et de le lier à un ou plusieurs registres. Ainsi, nous avons construit deux sous-ensembles principaux. Le premier consiste à relever des éléments factuels nécessaires à l'identité de la marque (nom et date de création) et à l'identification de la publicité (titre et année du (es) spot(s) et/ou visuel(s)). Le second répertorie tous les éléments constitutifs du positionnement communicationnel : scénario, personnages (âge, sexe, costume, attitude et comportement), mise en scène visuelle et sonore (éléments de décor, musique, ambiance) et éléments discursifs (voix off, slogan et signature du spot). L'objectif de la construction de cette grille est de repérer l'existence d'une stratégie de patrimonialisation publicitaire, d'en décortiquer les procédés et de relever les éléments invariants.

Il est à noter que l'analyse n'a pas porté sur l'ensemble des stratégies de communication mises en œuvre par une marque à travers le temps et à travers les supports médiatiques. Nous n'avons pas effectué d'analyse via un historique précis. Nous avons cependant eu recours, pour chacune des marques, à leur site institutionnel afin de valider leur date de naissance et l'historique de leurs spots télévisuels tels qu'elles le mettent en exergue. Compte tenu de I'histoire française de la publicité télévisée (autorisation des publicités commerciales depuis le $1^{\text {er }}$ octobre 1968), le travail s'est effectué sur une période 1970-1980 pour les plus anciennes jusqu'à 2017. Exceptée la marque Puget (1857), toutes les autres sont des marques créées au XXe siècle. Huit d'entre elles, parce qu'existantes avant 1968, ont d'abord communiqué via l'affichage ou les encarts presse. Les deux plus récentes (Hollywood (1972) et Petit Écolier (1983)) ont 
profité de l'essor de la publicité télévisuelle des années 1970-1990 avec, entre autres, une concentration des messages publicitaires sur un nombre restreint de chaînes de télévision (Tungate, 2009). Point commun entre elles : elles ont traversé le temps et choisi de le dire afin de valoriser le présent par le passé.

\section{2.}

\section{Une communication authentique et nostalgique}

Comment parlent-elles du passé et plus spécifiquement de leur propre passé publicitaire et quels symboles de leur " patrimoine publicitaire " mettentelles en avant ? Nous allons voir que les stimuli activés mettent en scène une représentation du passé articulée entre la nostalgie et l'authenticité.

\subsection{Entre longévité et modernité}

Le qualificatif de patrimoine, concept caractérisé par la continuité entre passé, présent, futur (Chastel, 1986 ; Davallon, 2006), suffit à évoquer une marque éternelle qui sera encore là demain. Le patrimoine n'englobe pas uniquement les formes matérielles (monuments, mobiliers, sites architecturaux) mais également les formes culturelles immatérielles. Ainsi naissent les archives télévisées permettant la patrimonialisation de matières audiovisuelles et plus spécifiquement celles publicitaires. Le concept de " patrimonialisation » apparaît pour interroger le processus qui conduit à conférer le statut de patrimoine. Ainsi, un objet jusque-là sans intérêt se patrimonialise grâce à un autre regard, un autre point de vue. Jean Davallon (2006) explicite les étapes du processus (trouvaille, authentification, exposition et transmission aux générations futures) et Philippe Le Guern, dans son analyse sur la patrimonialisation du rock, explique les trois moments induits entre « [...] l'identification des traces, leur conservation et, enfin, leur exploitation [...] » $(2012,12)$. Les marques, à la recherche d'une légitimité, s'inscrivent dans ces mouvements et s'auto-déclarent " patrimoniales ». Elles évoluent continuellement (réinvention permanente) mais gardent néanmoins le cap de leur signature, modelée au fil du temps en fonction des paradigmes culturels en vogue. L'authenticité, « [...] concept imprécis, aux facettes multiples et aux bornes fluides » (Tirloni, 2015, 507) inclut plusieurs signifiés rappelés par Valentina Tirloni : " [...] vérité, véridicité, véracité, sincérité, naturel, juste, exact, certifié conforme à l'original et, peut-être la plus intéressante, l'autorité » $(2015,508)$. Adaptée et adoptée par les marques dans une perspective marketing, l'authenticité est ici liée à la pérennité et à la singularité, à la sincérité et à la qualité car si l'histoire est importante les territoires aussi, autrement dit le local et la ruralité, la nature et le naturel et plus loin les responsabilités sociales et environnementales (Pecolo et Bahuaud, 2016). Les marques alimentaires adoptent facilement dans leurs communications ces deux facettes de l'authenticité, histoire et terroir, comme critères réconfortants Unmarked définie par mbahuaud. Le régionalisme fait partie des leviers d'innovation et 
reconsidérer une pratique ancestrale est un courant qui valorise un retour aux valeurs essentielles. Face aux crises alimentaires, les ingrédients, l'origine, le lieu de fabrication rassurent et la tradition (transmission d'un savoir-faire) est un argument fréquemment utilisé pour faire oublier les procédés industriels qui se cachent derrière la production (Fort et Fort, 2006). Interrogé par une enfant, le directeur d'une usine Mousline explique, dans un spot de 2017, en chanson et sur les mêmes notes de musique que celles des années 1970, la provenance de ses ingrédients : "Quand on fait de la purée Mousline, on est sûr de ce qu'il y a dedans. Beaucoup de pommes de terre cultivées dans les champs de Picardie ". La mise en scène débute dans une cuisine au sein de laquelle une maman apporte une assiette de purée à sa fille, se poursuit à la campagne lors de la récolte de pommes de terre par des tracteurs, pour finir avec le zoom sur le doigt que la petite fille trempe et lèche d'un air ravi. La marque Puget nous livre une saga servie par des personnages (Fernandel (de 2005 à 2014) et Michel Boujenah (depuis 2012)) aux accents méditerranéens sur fond de cigales et de lavande. Le spot de 2017 retrace I'histoire de la marque à travers trois scénettes chacune titrée chronologiquement : "Quelque part en Provence le 14 juin 1857 », " Au cœur de la Provence le 14 juin 1967 » et la dernière « Toujours en Provence le 14 juin 2017 ».

Authenticité et éternité sont donc ici les cartes communicationnelles favorites de ces marques patrimoniales qui usent d'une temporalité ouverte avec par exemple l'usage du " depuis... ", comme dans le cadre du spot de Puget «De table en table depuis 160 ans ». Visitons à présent cette autre modalité communicationnelle qui consiste à éveiller les sentiments nostalgiques d'une génération.

\subsection{De l'intemporel au générationnel}

Une génération est rattachée à une époque. Aussi, toute représentation d'un passé suffisamment récent est susceptible de mobiliser plusieurs formes de nostalgie. Il peut s'agir d'une nostalgie personnelle suscitant souvenirs d'enfants et d'adolescents et/ou d'une nostalgie collective réveillant un sentiment d'appartenance à une génération. Une nostalgie peut être également qualifiée $d^{\prime}$ ' historique " et peut être ressentie même par les plus jeunes qui n'ont pas vécu l'époque relatée. En effet, les plus jeunes la connaissent, notamment par le prisme de l'industrie médiatique (Lizardi, 2014), et bien souvent l'associent alors à un imaginaire positif. Toucher une génération spécifique (les générations dites Y, X ou baby-boomers ${ }^{5}$ (Strauss et Howe, 1991)) en utilisant ses marqueurs culturels, c'est également pouvoir réveiller chez leurs parents et grands-parents, une nostalgie de ces moments passés avec leurs enfants et petits-enfants d'alors.

5 La " génération $X$ " représente des individus nés entre 1960 et 1980 et les baby-boomers ceux nés entre 1943-1960 (Strauss et Howe, 1991). 
La nostalgie concilie des positions progressives, voire utopiques, ainsi que des attitudes régressives et mélancoliques (Pickering et Keightley, 2006). Comme le démontrent Maël Guesdon et Philippe Le Guern dans leur analyse sur les tendances rétro de la musique populaire (2014), cette ambivalence entre " renouvellement rétrospectif d'un idéal perdu » (Niemeyer, 2014, 13) et processus créatif est inhérente à la nostalgie (tension entre disparition et retour) et se retrouve dans les productions des industries culturelles. Conceptualisée par Hofer au XVII siècle, la nostalgie sera d'abord associée à une maladie mentale, une forme de mélancolie ou de dépression liée au lieu et/ou au temps lointain. Des travaux plus récents en psychologie démontrent la prégnance quotidienne de la nostalgie pour contrer nos humeurs négatives (Wildschut et al., 2006). Cette coloration plus positive est instrumentalisée dans le champ du marketing et de la communication qui présente la nostalgie comme une réaction affective douce-amère, une humeur mélancolique suscitée par l'évocation positive d'un passé regretté et idéalisé (Divard et Robert-Demontrond, 1997 ; Kessous et Roux, 2012). Rétrospectivement, la société d'il y a au moins 20 ans est toujours parée de toutes les vertus et la nostalgie, véhiculée par les productions médiatiques et culturelles, est alors simulée et construite (Weinstein, 2014) selon des intérêts économiques et commerciaux. Dans leurs travaux sur des publicités imprimées, Michael Pickering et Emily Keightley démontrent de quelle façon « la mémoire populaire est exploitée pour accroitre l'attrait des marchandises sur le marché " $(2014,88)$ et comment les sentiments et les valeurs nostalgiques peuvent être manipulés à travers une nostalgie régressive grâce à un procédé qu'ils nomment "retrotyping ", sorte " d'emballage consumériste du passé » $(2014,99)$. Ce dernier éloigne les éléments " indésirables » voire ambivalents du passé pour ne conserver que les plus joyeux, les plus idéalisés et embellis par les professionnels de la communication. Ces tactiques professionnelles n'empêcheraient pas, malgré tout, les récepteurs d'éprouver une authentique nostalgie.

Lorsque les marques de notre corpus mobilisent dans leur communication leur patrimoine, elles peuvent chercher à incarner le passé dans des périodes et des générations précises. Pour exemple, convoquer dans son argumentaire ou sa mise en scène un passé publicitaire suffisamment récent permet alors à une marque de s'adresser à toutes les générations encore en vie. Ainsi, des années 1970 aux années 1990, le vivier du marketing générationnel est large. Cette lecture générationnelle nous intéresse car ce sont souvent les codes d'une génération spécifique (sa jeunesse le plus souvent) ou l'évocation plus largement des générations antérieures qui servent à l'élaboration des scénarios publicitaires.

De 2009 à 2012, la marque de pâtes Panzani lance sa campagne « Panzani aussi », chantée sur l'air de la chanson « Félicie aussi » de Fernandel. Ce faisant, elle renvoie aux premières sagas publicitaires « Don Patillo » débutées en 1973 avec les pâtes, qui s'inspiraient de l'univers des Don Camillo interprétés dans les années 1950 par l'acteur Fernandel. La référence à ce dernier est donc double 
et différents niveaux s'imbriquent pour faire écho à différentes générations : celle qui a connu Fernandel dans sa jeunesse et celle qui se souvient de cette longue saga publicitaire " Don Patillo » (de 1973 à 2000). Une autre saga est relevée chez le concurrent Lustucru avec la cuisinière Germaine apparue pour la première fois en 1970 et personnage central de la saga « Germaine et les martiens " au milieu des années 1980. En 2010, la marque réalise un mini-film intitulé " le retour ». II (re)met en scène Germaine et les petits bonhommes verts. Des éléments invariants peuvent être relevés comme déclencheurs de la nostalgie, nous y reviendrons dans la partie suivante. En 1986, la marque "Caprice des Dieux " met en scène pour la première fois, un couple sur une plage et une voix off " on se fait un petit caprice ? Tous les deux ? ». En 2013, dans une publicité intitulée "Les sauveteurs " on retrouve le même décor et un scénario rappelant la série télévisée «Alerte à Malibu » diffusée de 1989 à 2001 et considérée comme la "cultissime série des années 90 ". Enfin, ces mêmes enfants et jeunes des années 1990 sont interpellés dans le spot "La Riposte » des Petit Écolier de Lu évoqué ci-dessus. C'est un clin d'œil à la saga 1985-2001 et sa signature "Ce n'est que pour les enfants » jouant sur l'opposition enfants/adolescents contre adulte voleur de gâteaux. Ces enfants, adultes aujourd'hui, prennent leur revanche en les dégustant, sous le nez des (nouveaux) enfants furieux et en affirmant "Y'a pas d'âge pour la Récré ! ".

Se greffe, dans ces exemples, un clin d'œil particulier (pas forcément repéré et/ou repérable par les plus jeunes) à la génération dite $X$ (registre générationnel), voire dans le cas de Panzani aux baby-boomers, et plus largement, à tous ceux ayant connu et retenu ces publicités d'il y a 20 ans. Ces retours publicitaires, via des personnages ou/et des scénarii réanimés, sont toujours d'abord, à destination des individus ayant connu les publicités originales. De quoi se souvenir, le temps d'un spot, du bon vieux temps.

\section{3. \\ Les éléments invariants : entre marqueurs culturels et registres sensoriels}

Mais qu'est-ce qui peut déclencher la nostalgie ? Vaste question à laquelle il est impossible de répondre de manière univoque car la nostalgie n'est pas qu'une mode ou une tendance confinée dans le champ publicitaire. "Elle est liée à une manière de vivre, d'imaginer et parfois d'exploiter ou de (re)inventer le passé, le présent et le futur [...] »(Niemeyer, 2014, 2). Néanmoins, dans les champ et corpus particuliers explorés ici, rappelons le lien très fort entre les écrits professionnels et les différents experts et cabinets conseils (Ohl, 2002). 
Comme le souligne Simona De lulio «Le domaine de la publicité se caractérise, en effet, par des échanges entre les travaux académiques et les réflexions des professionnels beaucoup plus fréquents et soutenus qu'il n'est d'ordinaire dans les sciences humaines et sociales [...]. La publicité est un dispositif marchand multiforme, diversifié et en évolution constante qui instrumentalise les travaux scientifiques mais, en contrepartie, ressort transformée par sa confrontation avec la science » $(2016,102)$. II n'est donc pas étonnant de repérer dans notre corpus publicitaire des procédés relatés par ailleurs dans les travaux en sciences de gestion. Ainsi, Ronan Divard et Philippe Robert-Demontrond exposent les résultats d'une étude anglo-saxonne portant sur l'identification de thèmes suscitant le plus la nostalgie $(1997,52)$ et pouvant ainsi être repris par les créatifs publicitaires. Cinq catégories de thèmes sont suggérées : les personnes importantes (par exemple les grands-parents) ; les objets ; les stimuli intangibles et représentatifs (arômes, musique etc.) ; les lieux et enfin les événements marquants (anniversaires, mariages, naissances...). D'autres recherches mettent également en avant les " facettes sociales " de la nostalgie (Wildschut et al., 2006,977 ) et soulignent l'importance des personnes proches (amis et famille) comme éléments déclencheurs tout comme les événements marquants et l'activation sensorielle (odeur ou goût par exemple).

Au-delà des thèmes sur lesquels les créatifs publicitaires peuvent travailler, certaines périodes du cycle de vie, parce qu'elles sont plus marquées par des discontinuités et des changements majeurs, sont plus à même de générer des sentiments nostalgiques. Il s'agit de l'adolescence et des premières années de l'âge adulte. Autrement dit, en fonction de leurs cibles, les publicitaires vont utiliser des stimuli correspondant à leurs années d'adolescents ou de jeunes adultes soit, en lien avec notre corpus, des individus nés entre 1960 et 1970. L'intérêt de l'approche générationnelle adoptée prend ici tout son sens en ce qu'elle permet d'associer marqueurs générationnels et procédés sonores, visuels et discursifs, réanimés par les publicitaires de manière plus ou moins discrète, plus ou moins directe.

\subsection{Des souvenirs sonores réactivés}

Nous avons déjà évoqué précédemment le spot publicitaire de Puget "De table en table depuis 160 ans » sorti en 2017, clin d'œil aux publicités anciennes. Outre la référence constante à la Provence, il est possible de repérer la jeune fille à vélo (transportant sur son porte-bagage une bouteille d'huile d'olive) scène déjà présente dès 1993. Mais c'est un autre élément sensoriel de cette même publicité « réanimée » qui attire notre attention. Nous pouvons en effet relever le recours en fond musical à la chanson "Tout l'amour que j'ai pour toi » de Dario Moréno (chanteur des années 1950). Interrogé dans la presse professionnelle, Romain Nouffert, chef de produit Puget chez Unilever relève la prégnance encore aujourd'hui de cette association Puget-Moreno : "Difficile d'entendre Dario Moreno sans penser à une jeune fille à bicyclette, sa jupe provençale fouettée par le vent de Porquerolles et la bouteille d'huile 
d'olive dépassant du panier à commissions [...]. Nous l'avons arrêtée depuis plus de dix ans, mais elle est encore citée spontanément ! »7.

Cette association chanson et sentiment nostalgique est également exploitée par des marques telles que Mousline (reprise de l'air et quelques paroles de la chanson des publicités depuis 1976 « Quand on fait de la purée Mousline, on est sûr de ce qu'il y a dedans »), mais aussi Les Produits Laitiers. Les premières publicités télévisées ont été conçues à partir de 1981 avec le refrain "Les produits laitiers sont nos amis pour la vie ", que l'on retrouve en 2015, non plus en signature de campagne mais intégré dans le schéma narratif des deux spots composant la campagne. Dans l'un, une petite fille demande à son oncle : "Dis tonton, tu me chantes les produits laitiers?", dans l'autre, une femme s'adresse à son mari "Dis chéri, tu me chantes les produits laitiers?", comme si la musique des produits laitiers était une référence familiale. Ou comment intégrer un refrain dans la culture médiatique populaire.

\subsection{Une reproduction de récits et de procédés visuels et discursifs}

Hollywood (ou Perrier pour " Hot Airs Baloons " en 2015) réaffirme son positionnement communicationnel, inchangé depuis des décennies, en réalisant un spot publicitaire reprenant tels quels les spots les plus marquants des décennies précédentes. Ainsi, en 2012 à l'occasion de ses 60 ans, la marque de chewing-gum rappelle à travers son spot "Hollywood chewing-gum, 60 ans et toujours la même fraicheur de vie " à la fois son âge et son héritage avec la même musique comme déclencheur de souvenirs.

Au-delà des personnages célèbres ou imaginaires, au-delà de la chanson, les publicitaires peuvent opter pour la reproduction d'un récit identique. Tel est le cas pour la marque Crunch dont la première publicité télévisuelle réalisée par Jean-Jacques Annaud remonte à 1970 avec le film «La campagne » et son " effet Crunch ». Quarante-deux publicités ont été diffusées de 1970 à 2003 avec pour point commun : I'explosion due au "chocolat qui croustille ». Après 9 ans de silence publicitaire, la marque est revenue en 2012 sur les petits écrans avec un nouveau spot, un nouveau protagoniste (le youtubeur Norman Thavaud), un slogan modernisé « Crunch ça déchire » mais l'explosion demeure comme élément invariant, véritable image de marque du chocolat.

Nous avons déjà évoqué le retour de Germaine chez Lustucru en 2010. Par rapport aux publicités des années 1980, Germaine demeure parée de sa charlotte, son tablier et son châle. Elle est en compagnie des martiens (représentation identique) qui réclament toujours en cœur la grand-mère « Germaine,

7 Fagès C., " Dg de Lesieur, Romain Nouffert a ressuscité Fernandel pour Puget, marque au sein de laquelle il débuta il y a 36 ans ", in emarketing. fr, 03 octobre 2017. Disponible sur http://www.e-marketing.fr/Thematique/ management-1090/Breves/-tete-Lesieur-Romain-nouffert-revient-ans-publicite-Puget-321418.htm 
Germaine... " en tapant sur la table. Si dans les spots des années 1980, Germaine voulait mais ne pouvait pas rentrer chez elle, dans celui-ci ce sont les martiens qui se retrouvent dans cette situation.

En 2010, la marque Lesieur commande à l'institut de sondage Sofres une étude sur son image. Il en ressort une forte association entre le slogan signature «Pas d'erreur c'est Lesieur » créé en 1986 (mais non réutilisé par la suite) et la marque. Aussi, en 2011 elle propose une nouvelle saga inspirée du spot de 1986 avec pour points communs : le format musical (paroles chantées), le montage (rapide avec des gros plans et des zooms), les couleurs vives (vert et bleu), les costumes (les robes et les jupes), les personnages (essentiellement féminins) et surtout ce slogan élément central de cette campagne, associé aux paroles « je veux tout ».

Pour conclure, nous pouvons relever, à travers les marques étudiées, que les quarantenaires actuels, enfants d'hier et parents aujourd'hui, sont des cibles idéales pour transmettre le produit à leurs propres enfants, permettant le renouvellement générationnel. Ne pas perdre une génération d'enfants, considérés comme les parents de demain, c'est continuer à communiquer dans le présent. C'est également s'adapter aux cultures actuelles, donc évoluer mais tout en gardant ses habitués, les anciens enfants et faire le pari sur leur pouvoir de prescription auprès de leur propre progéniture. En clair changer d'enfants (nouveautés) en gardant les parents (pointe nostalgique) et relier les générations. En opérant ainsi, ces publicités, parce qu'elles proposent ce récit reconnu, permettraient de constituer des références partagées (Fantin et Le Hégarat, 2016, 13), de générer un sentiment d'appartenance si ce n'est à une génération (car nous manquons de preuves), au moins à un groupe d'appartenance (Wildschut et al., 2006) et comme toute autre pratique médiatique de lier les générations (Le Guern, 2012, 30).

Nous avons pu constater que les entrées authenticité et nostalgie sont les terreaux favoris de cette forme de communication publicitaire. II suffit d'avoir franchi le cap d'un renouvellement générationnel (environ 20 ans), pour pouvoir jouer la carte nostalgique, de deux générations pour pouvoir devenir transgénérationnelle (environ 40 ans). Réveiller la fonction marchande initiale de l'artefact publicitaire ancien et de fait, ranimer un dispositif communicationnel éphémère questionne tout à la fois la place de la publicité dans notre société contemporaine (Baudrillard, 1986 ; Coccia, 2013) et le lien entre les mutations sociales, les imaginaires communicationnels des publicitaires (Soulages et Lochard, 1994) et les imaginaires générationnels (Balandier, 2012). 
Bibliographie

Angé O. (2015). « Le goût d'autrefois ". In Terrain [En ligne], $n^{\circ}$ 65, p. 34-51. Disponible sur http:// terrain.revues.org/15808 (page consultée le 03 novembre 2015).

Balandier G. (2012). Carnaval des apparences ou Nouveaux commencements?, Paris, Fayard.

Baudrillard J. (1986). La société de consommation : ses mythes, ses structures, Paris, Denoël.

Camus S. (2003). L'authenticité marchande perçue et la persuasion de la communication par l'authentification : une application au domaine alimentaire, Thèse de doctorat, Université de Bourgogne.

Chastel A. (1986). " La notion de patrimoine ». In Nora P. (dir.), Les lieux de mémoire, Paris, Gallimard, p. 405-450.

Coccia E. (2013). Le Bien dans les choses, Paris, Éd. Payot \& Rivages.

Cova B., Cova V. (2002). "Les particules expérientielles de la quête d'authenticité du consommateur $»$. In Décisions marketing, $\mathrm{n}^{\circ} 28$, p. 38-42.

Cova B., Louyot-Gallicher M., Bonnemaizon A. (2010). Marketing critique: le consommateur collaborateur en question, Paris, Lavoisier (EDF R\&D).
Davallon J. (2006). Le Don du patrimoine : Une approche communicationnelle de la patrimonialisation, Paris, Hermes Science-Lavoisier.

De Iulio S. (2016). Etudier la publicité, Grenoble, PUG.

Divard R., Robert-Demontrond P. (1997). " La nostalgie : un thème récent dans la recherche marketing ". In Recherche et Applications en Marketing, vol. 12, $n^{\circ} 4$, p. 41-61.

Dordor X. (1993). La presse professionnelle. Mieux la connaître pour mieux l'utiliser, Paris, Dunod.

Fantin E., Le Hégarat T. (2016). "L'Âge d'or ». In Le Temps des médias, $n^{\circ} 27$, p. 5-15.

Fort F., Fort F. (2006). « Alternatives marketing pour les produits de terroir ". In Revue française de gestion, vol. 162, n 3 , p. 145-159.

Guesdon M., Le Guern P. (2014). «Retromania : Crisis of the Progressive Ideal and Pop Music Spectrality ». In Niemeyer K. (dir.), Media and nostalgia. Yearning for the past, present and future, Basingstoke, Palgrave Macmillan, p. 70-80.

Hartog F. (2003). Régimes d'historicité : présentisme et expériences du temps, Paris, Seuil.

Kessous A., Roux E. (2012). « Nostalgie et management des marques : approche sémiotique ». In Management \& Avenir, vol. 54, n 4, p. 15-33. 
Le Guern P. (2012). « Un spectre hante le rock... L'obsession patrimoniale, les musiques populaires et actuelles et les enjeux de la 'muséomomification' ». In Questions de communication, $\mathrm{n}^{\circ} 22$, p. 7-44.

Niemeyer K. (2014). Media and nostalgia. Yearning for the past, present and future, Basingstoke, Palgrave Macmillan.

Nussbaum P., Evéquoz G. (2014). C'était mieux avant. Le syndrome du rétroviseur, Paris, Favre Pierre-Marcel Eds.

Ohl F. (2002). « La construction sociale des figures du consommateur et du client ". In Sciences de la Société, n 56, p. 24-41.

Pecolo A., Bahuaud M. (2016). «Patrimoine historique et publicitaire : la communication se saisit du passé ". In Communiquer, $\mathrm{n}^{\circ} 16$, p. 65-83.

Pecot F., De Barnier V. (2017). "Patrimoine de marque : le passé au service du management de la marque ". In Recherche et Applications en Marketing, vol. 32, $n^{\circ}$ 4, p. 77-96.

Pickering M., Keightley E. (2006). "The modalities of Nostalgia ». In Current Sociology, vol. 54, n 6, p. 919-941.

Pickering M., Keightley E. (2014). " Retrotyping and the Marketing of Nostalgia ». In Niemeyer K. (dir.), Media and nostalgia. Yearning for the past, present and future. Basingstoke, Palgrave Macmillan, p. 83-94.
Reynolds S. (2012). Retromania. Comment la culture pop recycle son passé pour s'inventer un futur, Marseille, Editions Le mot et le reste.

Soulages J.-C., Lochard G. (1994). "Les imaginaires de la parole télévisuelle : permanence, glissements et conflits ". In Réseaux, n63, p. 13-38.

Strauss W., Howe N. (1991). Generations. The History of America's Future, New-York, Quill William Morrow.

Tungate M. (2009). Le monde de la pub. Histoire globale (et inédite) de la publicité, Paris, Dunod.

Urde M., Greyser S. A., Balmer J. M. T. (2007). «Corporate brands with a heritage ». In Journal of Brand Management, vol. 15, $n^{\circ} 1$, p. 4-19.

Weinstein D. (2014). « La nostalgie construite ». In Volume ! [En ligne], vol. 11:1, n² 2, p. 19-36. Disponible sur http://journals. openedition.org/volume/4190 (page consultée le 01 octobre 2015).

Wildschut T., Sedikides C., Arndt J., Routledge C. (2006). « Nostalgia : Content, Triggers, Functions ». In Journal of Personality and Social Psychologie, vol. 91, $n^{\circ}$ 5, p. 975-993. 
